THE JOURNAL OF

PHYSICAL
CHEMISTRY
L E T T E R S

\title{
Energetics of ion transport in a peptide nanotube Supplementary information
}

\author{
François Dehez, Mounir Tarek and Christophe Chipot* \\ Equipe de dynamique des assemblages membranaires, Unité mixte de recherche CNRS/UHP 7565, Nancy Université, \\ BP 239, 54506 Vandœuvre-lès-Nancy cedex, France
}

Received: August 2, 2007

\begin{abstract}
Description of the system. The peptide nanotube consisted of eight stacked cyclo $[\underline{\mathrm{LW}}]_{4}$ units, where underlined letters denote D-amino acids. The sequence of alternated D- and $\mathrm{L}-\alpha$-amino acids guarantees that all cyclic peptide are reasonably flat, with their side chains pointing outwards. The aqueous environment consisted of a bath of 2,506 water molecules. This corresponds to an equilibrated box of dimensions $40 \times 40 \times 55 \AA^{3}$. The model membrane was formed by 48 palmitoyloleylphosphatidylcholine (POPC) units in equilibrium with 1,572 water molecules. placed in a simulation cell of dimensions $36 \times 41 \times 79 \AA^{3}$.
\end{abstract}

Molecular dynamics simulations. All molecular dynamics (MD) simulations were carried out in the isothermalisobaric ensemble, using the program NAMD ${ }^{1}$ and the all-atom CHARMM27 force field. ${ }^{2}$ The temperature and the pressure were maintained at $300 \mathrm{~K}$ and 1 atm, respectively, employing Langevin dynamics and the Langevin piston method. ${ }^{3}$ Longrange electrostatic forces were taken into account by means of the particle mesh Ewald (PME) approach. ${ }^{4,5}$ The equations of motion were integrated with the r-RESPA multiple time-step propagator $^{6}$ and a $2-\mathrm{fs}$ time step. All chemical bonds between heavy and hydrogen atoms were constrained to their equilibrium distance. For consistency between the simulations in the bulk aqueous medium and in the membrane environment, the distance separating the nitrogen atoms of the amino moieties and the carbon atoms of the carbonyl groups pertaining to adjacent cyclic peptides was restrained to its equilibrium value by means of soft harmonic potentials. ${ }^{7}$

Free energy calculations. To investigate ion conduction in the peptide nanotube, the reaction coordinate $(\mathrm{RC}), \xi$, was chosen as the distance separating the ion from the center of mass of

\footnotetext{
*To whom correspondence should be addressed. E-mail: Christophe.Chipot@edam.uhp-nancy.fr
}

the hollow cylindrical structure, projected onto its longitudinal axis. Variation of the free energy, $\Delta G(\xi)$, along that direction was determined using the adaptive biasing force (ABF) method, ${ }^{8}$ which relies upon the integration of the average force acting on the RC, obtained from unconstrained MD simulations. ${ }^{9}$ The free energy derivative, $\mathrm{d} G(\xi) / \mathrm{d} \xi$, is estimated locally throughout the simulation, thus providing a continuous update of the biasing force. When applied to the system, this bias generates a Hamiltonian bereft of a net average force along the RC. Consequently, all values of the reaction coordinate are sampled with an equal probability, thereby improving significantly the accuracy of the computed free energies.

Mapping electric properties. The three-dimensional maps of the electrostatic potential characterizing the peptide nanotube immersed in the bulk aqueous medium and in the model membrane were generated by solving numerically the Poisson equation. This calculation was performed employing the dedicated PMEPot $^{10}$ module of the visualization program VMD. ${ }^{11}$ Instantaneous values of the electrostatic potential were averaged over 500-ps segments of the complete MD trajectories. The first derivative of the electrostatic potential was evaluated using OpenDx [http://www.opendx.org], an open-source visualization software package, to yield the three-dimensional maps of the electric field. The electric properties of the molecular assemblies described in this contribution were visualized with OpenDX and the CMSP Chemistry module. ${ }^{12}$

In addition to the electrostatic potential mapped in the absence of the cation, the same quantity has been determined when the sodium ion was located in the midst of the hollow tubular structure, both in the aqueous and in the membrane environments. Not too unexpectedly, Figure 1 reveals that the electrostatic potential is markedly distorted in the vicinity of the ion. Yet, the topology of the electrostatic landscape remains globally unaltered at the extremities of the peptide nanotube.

The present simulations admittedly only involved a single 

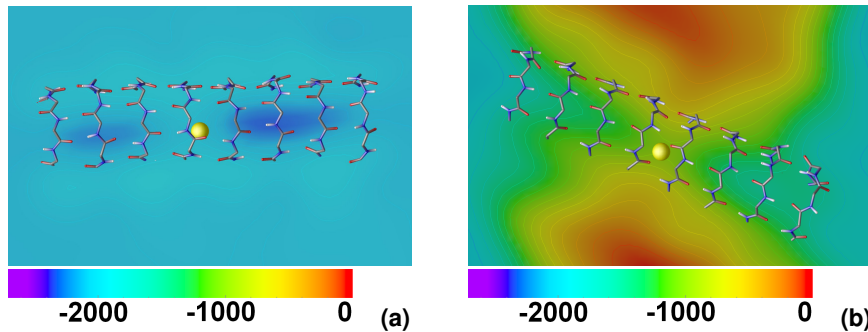

Figure 1. Cross-sectional view of the three-dimensional map of the electrostatic potential determined in the presence of the sodium ions when the synthetic channel is immersed in an aqueous medium (a) and in a model membrane (b). Units are given in $\mathrm{mV}$.

synthetic channel with a given pore radius. Yet, tubular structures formed by larger, twelve $\alpha$-amino-acid cyclic peptides have been found to yield similar electrostatic signatures, either in water or in a membrane environment [results not shown here].

Coordination of the cation. Coordination motifs of the sodium ion in the hollow tubular structure has been examined as a function of its position along the $\mathrm{RC}, \xi$. When located in the in- or $\alpha$-plane of the cyclic peptides, the cation is chelated by an average of five water molecules. Hopping to the midplane materialized between two consecutive rings is accompanied by a partial dehydration of the ion, which is compensated by the transient formation of non-covalent bonds with the participating carbonyl groups of the synthetic channel.

Removal of the mouth of the pore from the surface of the membrane. To appreciate the role played by the position of the mouth of the pore with respect to the water-membrane interface, a longer, partially immersed tubular structure was considered. This alternative synthetic channel is formed by eleven stacked cyclic peptides, three among which protrude above the surface of the lipid bilayer. One nanosecond of MD trajectory was generated, from whence the electrostatic potential and the electric field were mapped. It is apparent from Figure 2, that when the mouth of the peptide nanotube is appreciably far from the water-membrane interface, an electrostatic barrier and, hence, a net electric force arises, reminiscing the case of the synthetic channel immersed in a bulk aqueous environment.

Free diffusion of the cation. The position-dependent selfdiffusion of the sodium ion in the hollow tubular structure has been determined. A convenient, concise expression for $D(z)$ was derived by Hummer in the framework of an overdamped harmonic oscillator: ${ }^{13}$

$$
D(\xi=\langle\xi\rangle)=\frac{\operatorname{var}(\xi)}{\tau_{\xi}}
$$

Here, $\operatorname{var}(\xi)=\left\langle\xi^{2}\right\rangle-\langle\xi\rangle^{2}$ is the variance of the time-series of the $\mathrm{RC}$, and $\tau_{\xi}$ is its characteristic time:
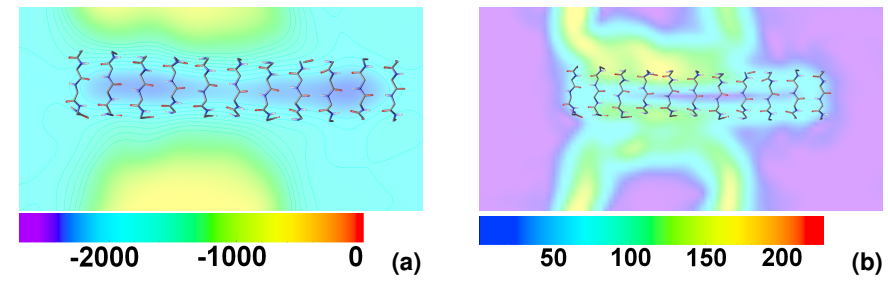

Figure 2. Cross-sectional view of the three-dimensional map of the electrostatic potential (a) and the electric field (b) determined for a synthetic channel formed by eleven stacked cyclo[ $[\mathrm{LW}]_{4}$ peptide units and immersed partially in a POPC lipid bilayer. Units are given in $\mathrm{mV}$ and $\mathrm{mV} / \AA$, respectively.

$$
\tau_{\xi}=\frac{\int_{0}^{+\infty}\langle\delta \xi(t) \delta \xi(0)\rangle \mathrm{d} t}{\operatorname{var}(\xi)}
$$

where $\delta \xi(t)=\xi(t)-\langle\xi\rangle$. The autocorrelation function of the RC was evaluated locally at ten different values of $\xi$ (see Figure 3) by sampling configurations in a quasi-harmonic well formed by a single bin $0.01 \AA$ wide supplemented by energy barriers $10 \mathrm{kcal} / \mathrm{mol} / \AA ̊ \mathrm{~A}$ high. Langevin dynamics was performed with a friction term of $10 \mathrm{ps}^{-1}$. At each selected point of the free energy landscape, an independent trajectory of up to $2 \mathrm{~ns}$ was generated to estimate the diffusion properties of the cation.

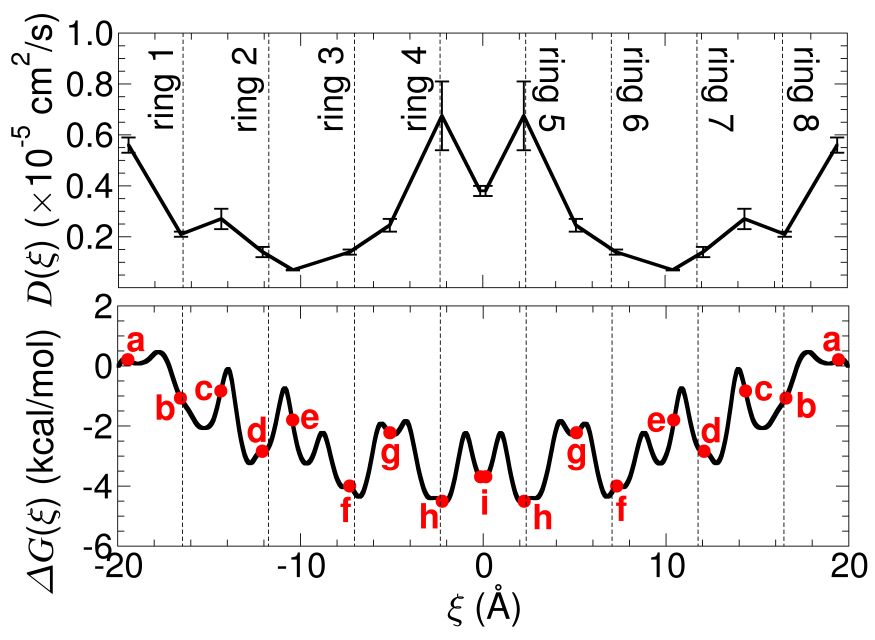

Figure 3. Position-dependent self-diffusion of the sodium ion in the synthetic channel embedded in a fully hydrated POPC bilayer. The free diffusion coefficients were inferred from local measures of the autocorrelation function of the RC, $\xi$, at different values of the latter - viz. points a to $\mathbf{j}$. Error bars were estimated from the uncertainty on $\tau_{\xi}$.

As a basis of comparison, the self-diffusion coefficient of the sodium ion was estimated in a bulk aqueous environment, from the Einstein relationship and the mean-square displacement ${ }^{14}$ and by applying equation (1) - viz. 2.0 and $1.7 \times$ $10^{-5} \mathrm{~cm}^{2} / \mathrm{s}$, respectively. Figure 3 reveals that diffusion is by and large enhanced near the center of the peptide nanotube, which corresponds to the lowest regions of the curved free energy surface. It is worth noting that the cation diffuses approxi- 
mately four times faster between the two central cyclic peptides than between either the second and the third, or the sixth and the seventh. Furthermore, even $c a .3 \AA$ above the first and the last peptide rings, the friction exerted onto the sodium ion is still significant. This environment is, therefore, distinct from that of bulk water, which is not completely surprising in view of the electric field landscape.

\section{References and Notes}

(1) J. C. Phillips, R. Braun, W. Wang, J. Gumbart, E. Tajkhorshid, E. Villa, C. Chipot, L. Skeel, R. D. Kalé, and K. Schulten, J. Comput. Chem., 2005, 26, 1781-1802.

(2) A. D. MacKerell Jr., D. Bashford, M. Bellott, R. L. Dunbrack Jr., J. D. Evanseck, M. J. Field, S. Fischer, J. Gao, H. Guo, S. Ha, D. Joseph-McCarthy, L. Kuchnir, K. Kuczera, F. T. K. Lau, C. Mattos, S. Michnick, T. Ngo, D. T. Nguyen, B. Prodhom, W. E. Reiher III, B. Roux, M. Schlenkrich, J. C. Smith, R. Stote, J. Straub, M. Watanabe, J. Wiórkiewicz-Kuczera, D. Yin, and M. Karplus, J. Phys. Chem. B, 1998, 102, 3586-3616.

(3) S. E. Feller, Y. H. Zhang, R. W. Pastor, and B. R Brooks, J. Chem. Phys., 1995, 103, 4613-4621.

(4) T. A. Darden, D. M. York, and L. G. Pedersen, J. Chem. Phys., 1993, 98, 10089-10092.

(5) U. Essman, L. Perera, M. Berkowitz, T. Darden, H. Lee, and L. G. Pedersen, J. Chem. Phys., 1995, 103, 8577-8593.

(6) M. E. Tuckerman, B. J. Berne, and G. J. Martyna, J. Phys. Chem. B, 1992, 97, 1990-2001.

(7) D. Asthagiri and D. Bashford, Biophys. J., 2002, 82, 1176-1189.

(8) E. Darve and A. Pohorille, J. Chem. Phys., 2001, 115, 9169-9183.

(9) J. Hénin and C. Chipot, J. Chem. Phys., 2004, 121, 2904-2914.

(10) A. Aksimentiev and K. Schulten, Biophys. J., 2005, 88, 3745-3761.

(11) W. Humphrey, A. Dalke, and K. Schulten, J. Molec. Graphics, 1996, 14, 33-38.

(12) R.E. Gillilan and F. Wood, Comput. Graph., 1995, 29, 55-58.

(13) G. Hummer, New J. Phys., 2005, 7, 34.

(14) A. Einstein, Annalen der Physik, 1905, 17, 549-560. 